\title{
Ecocardiografía transesofágica (ETE) durante cirugía no cardíaca
}

\author{
MARÍA CAROLINA CABRERA S. ${ }^{1}$
}

\section{La primera pregunta que podrían hacerse los lec- tores es ¿por qué? y ¿para qué? utilizar ecocar- diografía durante una cirugía no cardíaca}

a idea es que luego de leer esta revisión, ustedes estimados lectores, tengan su propia respuesta e

impresión de este tema.

La prolongación en la expectativa de vida de la población, ha redundado en que los pacientes quirúrgicos sean de mayor edad y portadores de patologías cardiovasculares crónicas y complejas.

Desde el punto de vista fisiopatológico se produce una severa alteración en la distensibilidad del tejido miocárdico y el ventrículo izquierdo se torna rígido, lo que es más difícil de manejar desde el punto de vista anestesiológico, dado que estos pacientes toleran mal las sobrecargas de volumen, la taquicardia y el aumento de la postcarga. También se ha visto que muchos de estos enfermos tienen su función diastólica alterada, incluso a pesar de tener función sistólica normal (hasta 40\% tienen diástole alterada con sístole conservada). Generalmente se encuentran en buenas condiciones basales y realizan una vida razonablemente normal, pero la reserva funcional de sus órganos está disminuida. Así frente a situaciones de stress, como es una cirugía, presentan descompensaciones perioperatorias. Se ha demostrado que una buena vigilancia durante la cirugía permite diagnosticar y manejar eventos no deseados y así disminuir la morbimortalidad postoperatoria.

Entonces de aquí surge la segunda pregunta: ¿Cómo vigilo, cómo monitorizo a estos pacientes?

En el año 1971, los doctores Swan y Ganz' describieron un catéter con un balón en su punta que permitía estimar las presiones de las cavidades cardíacas derechas y de la arteria pulmonar. Este catéter fue y es aún en la actualidad ampliamente utilizado, pero, ¿Las presiones corresponden a volúmenes?

¿Qué pasará en el miocardio con alteraciones de la distensibilidad?
Estos son justamente a los pacientes que nos enfrentamos en nuestra práctica clínica diaria. Enfermos portadores de diferentes tipos de cardiopatías, isquémica, hipertensiva, diabética, de larga evolución. Ellos se caracterizan por tener ventrículos izquierdos (VI) muy rígidos con una respuesta poco predecible los cambios de volumen.

En un estudio muy interesante realizado por Osman y sus colaboradores ${ }^{3}$, publicado en el Critical Care de 2007, se demostró que las presiones de llenado no eran capaces de predecir la respuesta del paciente al volumen. Es más, dividieron a los pacientes en dos grupos: los respondedores y los no respondedores a aportes de volumen y no se encontró correlación alguna entre los valores de presión y el volumen infundido.

Paul Marik y sus colaboradores ${ }^{5}$ realizaron una revisión sistemática de la literatura, y del análisis de más de 800 enfermos, no se logró demostrar correlación alguna entre el volumen y la presión.

Entonces preguntas simples, tales como:

¿Cómo está la volemia?

¿Tiene isquemia miocárdica?

¿Qué efectos tienen los fármacos vasoactivos en el miocardio?

Tienen respuestas muy difíciles.

En este contexto aparece la ecocardiografía tanto transtorácica como transesofágica, como una herramienta de monitorización mínimamente invasiva, que no interfiere con el campo quirúrgico y que es relativamente sencilla de instalar e interpretar, una vez que el anestesiólogo está entrenado ${ }^{2,4}$.

La ecocardiografía permite volver a tener un pensamiento fisiológico cardiovascular. El volumen de fin de diástole medido ecográficamente, sería más útil que la presión para evaluar la precarga ventricular, esta última depende más de la longuitud de la fibra miocárdica que de la presión en la cavidad izquierda. Por lo tanto es más seguro estimarla por el volumen que por la presión

Los beneficios de la ecocardiografía en cirugía no cardiaca están aún en investigación, dado que esta

Profesor auxiliar de Anestesiología

Universidad de Valparaíso, sede Hospital Fach. 
valoración no siempre es fácil de lograr6-9. Se trata de pacientes críticos y complejos, por su edad, sus patologías y cuadro clínico. No es el objetivo de este escrito, comparar el catéter de arteria pulmonar con la ecocardiografía, ya que ambos tipos de monitorización son completamente diferentes, pero debe pensarse en las limitantes con que se trabaja y en los aportes que puede tener la implementación de una nueva tecnología como la ecocardiografía para el monitoreo intraoperatorio de una cirugía no cardíaca.

Según las últimas guías norteamericanas, los pacientes con indicación de monitoreo electivo con ecocardiografía durante cirugía no cardíaca, serían aquellos portadores de cardiopatías en los que se piensa puedan ocurrir complicaciones hemodinámicas, pulmonares ${ }^{11}$. Los pacientes cardiópatas pueden ser coronarios, enfermos con antecedentes de falla ventricular y/o arritmias, pacientes valvulópatas y portadores de hipertensión pulmonar. El objetivo de la utilización de la Ecocardiografía transesofágica (ETE) es la monitorización directa y en tiempo real del corazón y sus grandes vasos, para así realizar un diagnóstico precoz y guiar la terapia con drogas vasoactivas, betabloqueadores y fluidos siguiendo las imágenes y mediciones del miocardio y cavidades cardíacas $^{10}$.

Existen diferentes parámetros ecocardiográficos que evalúan la función miocárdica intraoperatoria como son la fracción de eyección (FE) y el llenado del VI. La FE (Figura 1) es un buen índice de función sistólica, la cual, cuando está disminuida se correlaciona con un mayor riesgo de eventos luego de una cirugía no cardíaca. Las velocidades del llenado ventricular izquierdo evaluadas con Doppler (Figura 2) representan la función diastólica. La primera onda $\mathrm{E}$ (early) corresponde al llene temprano del VI y la onda A (auricular)

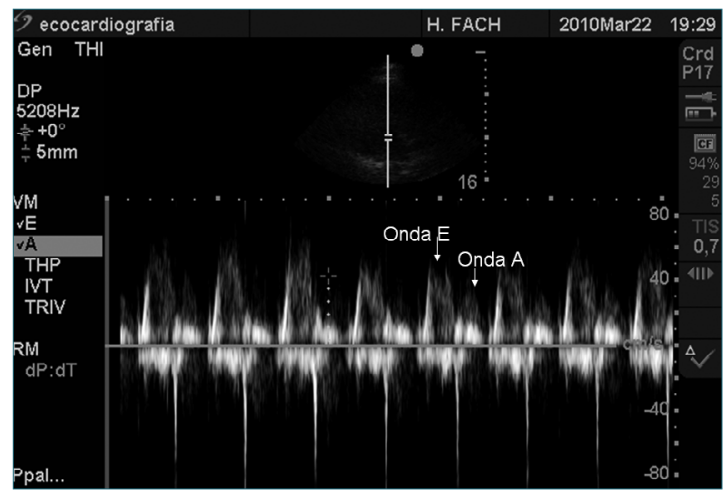

Figura 2. Función diastólica. Onda $E=$ = llene rápido; Onda A = sístole auricular.

corresponde a la sístole auricular. También se ha visto que la disfunción diastólica se asocia con un mayor número de eventos hemodinámicos postoperatorios, como hipotensión e hipertensión ${ }^{11-17}$. La Ecocardiografía también permite calcular el gasto cardíaco (GC), parámetro reconocido como un muy buen índice de función cardíaca global y predictor de complicaciones perioperatorias.

Además, con el uso de doppler tisular se ha producido un significativo avance para lograr una adecuada evaluación de la diástole del VI (Figura 3) y de la función global del corazón y ya se están investigando parámetros como el índice E/e', que es un predictor de la presión de fin de diástole del VI.

Entonces podemos enfrentarnos a dos escenarios posibles:

1.- Ecocardiografía intraoperatoria "electiva" en casos con antecedentes cardiovasculares importan-

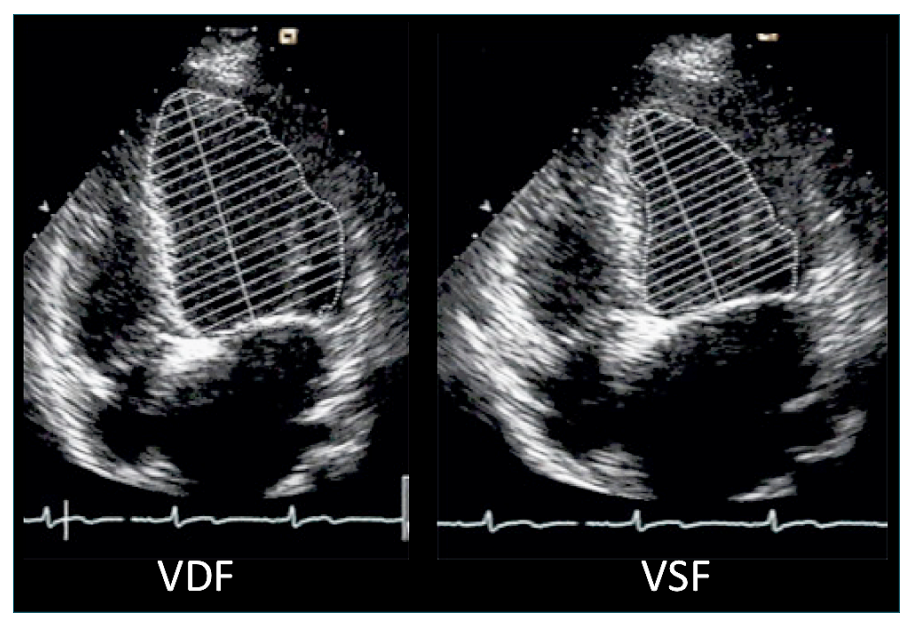

Figura 1. Fracción de eyección (FE). VDF = volumen diastólico final, VSF = volumen sistólico final. Para calcular

$$
F E=\frac{V F D-V D S}{V F D}
$$


tes y/o en que se espere un sangrado importante. 2.- Ecocardiografía intraoperatoria de "emergencia" en caso de un compromiso hemodinámico severo, en que no ha habido respuesta a terapia con volumen y vasopresores.

Algunos ejemplos donde la Ecocardiografía electiva puede ser útil:

\section{Cirugía vascular}

Una de las indicaciones más frecuentes para monitorizar con Ecocardiografía es durante el intraoperatorio de grandes cirugías vasculares, como por ejemplo aneurismas de aorta. Está bien demostrado que los pacientes sometidos a cirugía vascular mayor tienen mayor riesgo de presentar isquemia miocárdica perioperatoria. La sensibilidad y especificidad de la Ecocardiografía para la detección de nuevos eventos isquémicos es alta, pero la gran utilidad de la ETE está en que no sólo permite detectar episodios de isquemia intraoperatoria, sino que también permite manejar los volúmenes durante el clampeo aórtico y ajustarlos al momento de desclampear. También es útil para evaluar el efecto de fármacos como betabloqueadores, y en el caso de indicar fármacos vasoactivos rápidamente es posible visualizar sus efectos en la contractilidad miocárdica ${ }^{18}$. Otra virtud de la ecocardiografía es la detección de la hipovolemia, uno de cuyos signos característicos es "el beso de los músculos papilares" (Figura 4).

\section{Cirugía ortopédica}

Existe un grupo importante de pacientes añosos y portadores de múltiples patologías, entre ellas patología cardiovascular relevante, que son sometidos a procedimientos no electivos como fracturas de cadera donde existe riesgo de sangrado y embolización durante la cirugía.

También muchos enfermos son sometidos a cirugías que presentan gran riesgo de sangrado, por ejemplo, escoliosis.

En estos pacientes claramente la visualización del material embolizado permitirá realizar el diagnóstico y así decidir la mejor terapia, cuya respuesta dependerá de la magnitud de la carga embolizada y de su reserva funcional cardiopulmonar (Figura 5).

\section{Cirugía abdominal, renal y urológica}

Muchos de estos pacientes son también añosos y portadores de patología crónica cardiovascular. La detección de isquemia intraoperatoria con Ecocardiografía provocará cambios en la conducta y plan anestésico tanto intraoperatorio como postoperatorio,

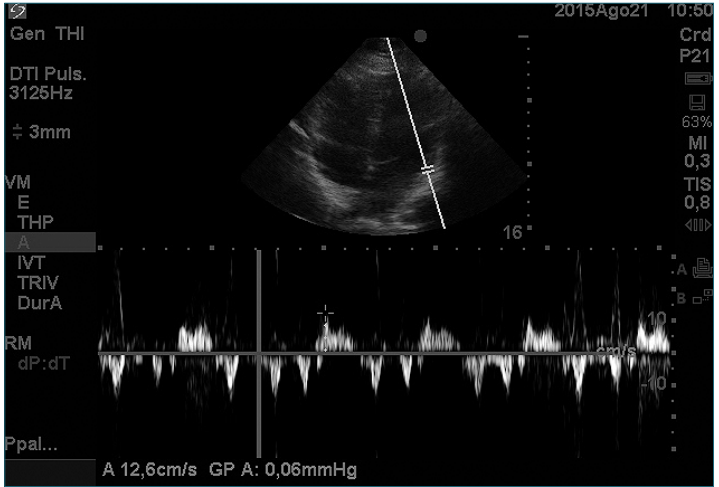

Figura 3. Doppler tisular.

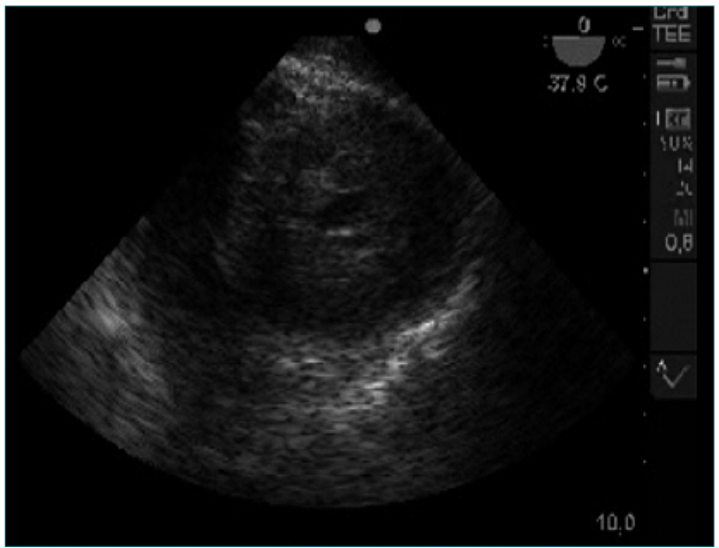

Figura 4. Visión de eje corto de VI donde músculos papilares se "besan".

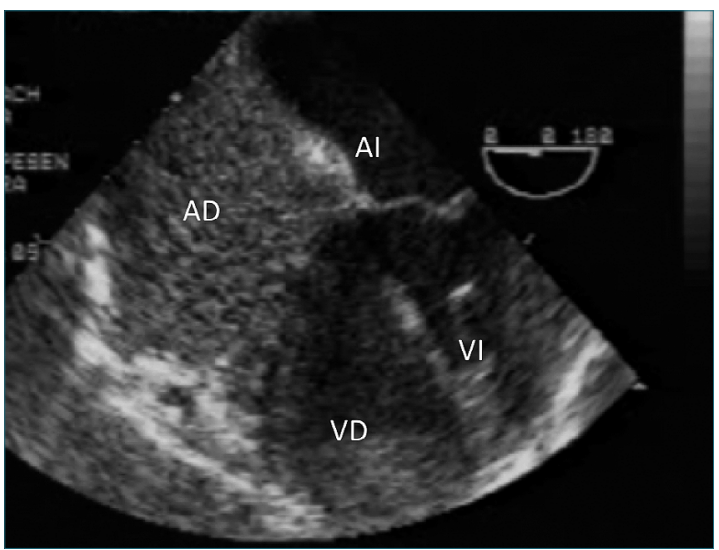

Figura 5. Embolia durante cirugía traumatológica. 


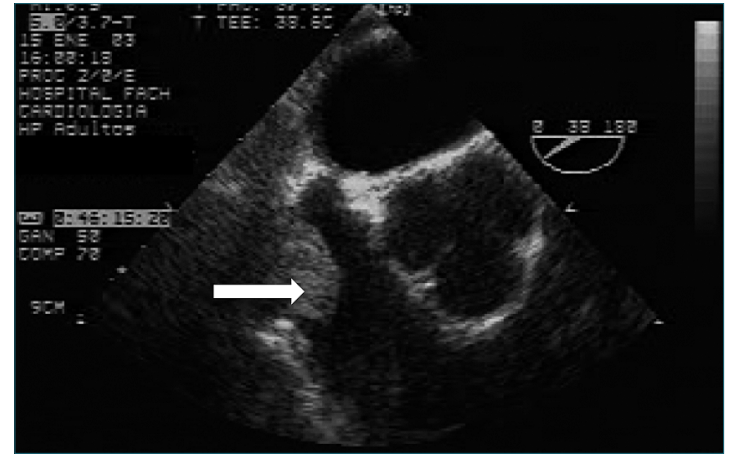

Figura 6. Hipernefroma que comprometía vena cava inferior hasta $A D$ donde se ve la flecha.

sugerirá el derivar al paciente a unidades de mayor complejidad e iniciar precozmente anticoagulantes y eventualmente realizar estudio de la anatomía coronaria en hemodinamia (Figura 6).

Existen cirugías en las que eventualmente pueden haber grandes cambios de volumen como prostatectomías radicales o tumorectomías renales. En el caso de cirugía de hipernefromas la ETE permite evaluar el grado de compromiso tumoral y si éste ha alcanzado las cavidades cardíacas derechas como se observa en la Figura 6.

Otra indicación muy interesante es durante la resección de un feocromocitoma, estos son pacientes en que la volemia es muy difícil de estimar y donde existen claramente dos tiempos quirúrgicos antes y después de la resección de éste.

\section{Neurocirugía}

Una de las primeras indicaciones de ETE durante cirugía no cardíaca fue la neurocirugía en posición sentada. Con el transductor instalado en esófago medio para monitorizar las cavidades cardíacas derechas, es posible visualizar la llegada de aire a estas cavidades. En la Figura 7 se observa el caso de un paciente en que la magnitud de la embolización fue muy grande y la detección de ésta por ecocardiogafía fue más precoz que el cambio de la curva de capnografía y el deterioro de la hemodinamia.

\section{Trauma}

En el trauma torácico agudo la ETE es una herramienta de alta utilidad para evidenciar disección traumática de aorta, contusiones miocárdicas y lesión de grandes vasos. También se ha demostrado su eficiencia en el politraumatizado grave, no sólo para monitorizar su hemodinamia, sino que también para realizar nuevos diagnósticos, como taponamiento cardíaco y lesio-

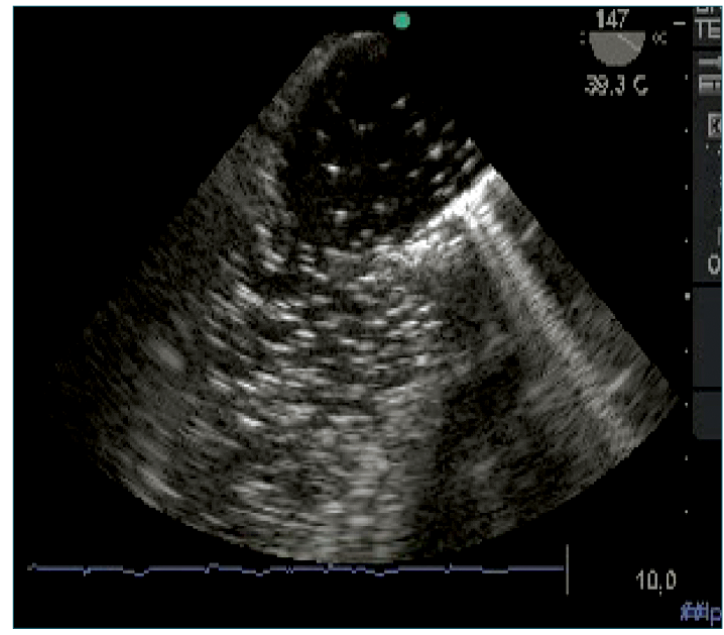

Figura 7. Embolia aérea.

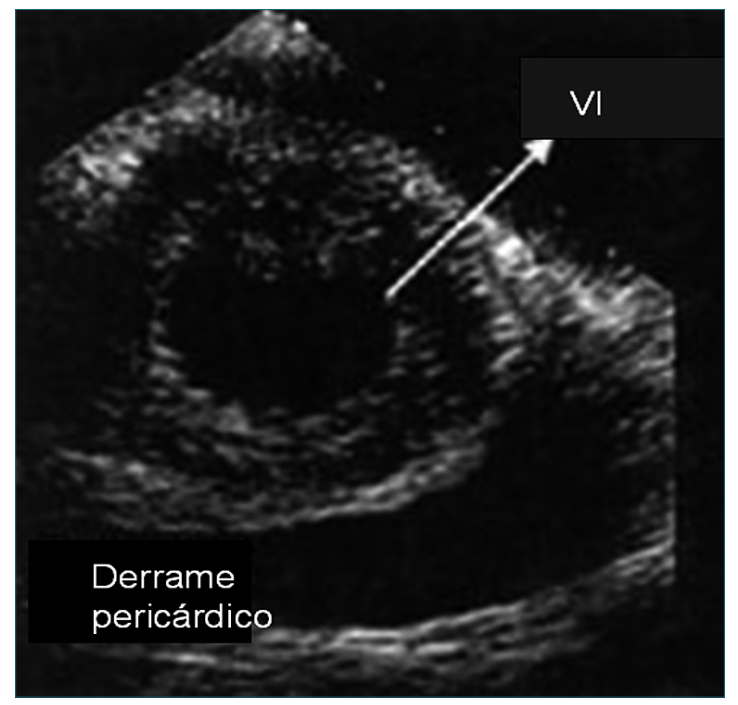

Figura 8. Derrame pericárdico.

nes de miocardio (Figura 8). Se ha utilizado la ecografía tanto transesofágica como transtorácica para monitorización de maniobras de resucitación cardiopulmonar y ha demostrado ser una herramienta efectiva.

\section{Segundo escenario: El "descalabro" hemodinámico}

El uso de la ecocardiografía también está indicado en cirugías no cardíacas en las que ocurre un severo compromiso hemodinámico que no responde al tra- 
tamiento convencional. En estos casos, independiente de las patologías de base del paciente, se ha demostrado la alta utilidad de la eco para lograr un diagnóstico etiológico y así manejar la causa del deterioro de la hemodinamia. Además su uso permite manejar y guiar la terapia que se indique al paciente, el uso de volumen, y el efecto en el miocardio de las diferentes drogas vasoactivas ${ }^{18-22}$.

Es importante tener en cuenta que hipotensión no es sinónimo de hipovolemia. No siempre el manejo de una hipotensión es la administración de volumen. Existen otras causas como isquemia miocárdica masiva, taponamiento pericárdico y/o movimiento sistólico anterior de la velo anterior de la válvula mitral (Figura 9).

Sin duda que esta técnica de monitorización semiinvasiva ofrece ventajas y tiene grandes áreas de desarrollo, pero también presenta desventajas, como el costo de equipamiento y la necesidad de tener una formación adecuada, "se ve lo que se sabe".

Uno de los problema de la ETE es el sobrediagnóstico o el subdiagnóstico por falta de experiencia y/o entrenamiento del operador. En Norteamérica y Europa la formación del anestesiólogo para realizar ecocardiografía ha sido dividida en niveles básico y avanzado. En el nivel básico el anestesiólogo debe ser capaz de reconocer todas las estructuras cardíacas, diagnosticar isquemia miocárdica, evaluar la hemodinamia y función ventricular. Se recomienda para esto un mínimo de 150 exámenes. Es muy importante que el operador reconozca sus limitaciones, no aventure

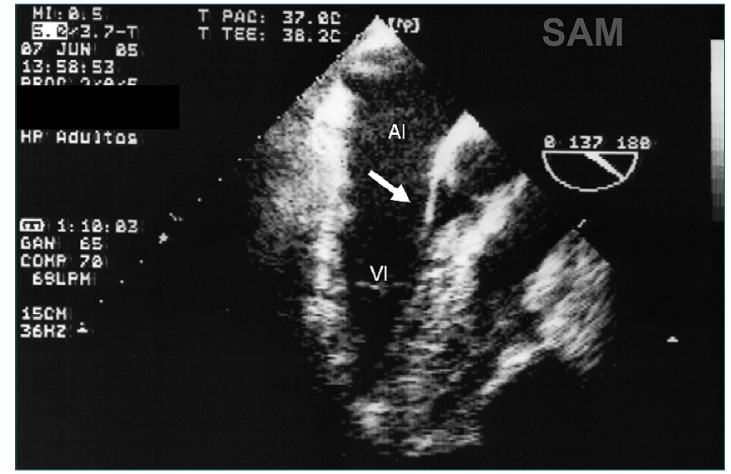

Figura 9. SAM (Sistolic anterior movement) de válvula mitral.

diagnósticos y solicite ayuda precozmente En la etapa más avanzada deberá, además, manejar Doppler para mediciones de gradientes y áreas, diagnosticar disección de aorta, endocarditis y trombos, y para esto se requiere de al menos 300 exámenes ${ }^{23-24}$.

En conclusión, la ETE durante el intraoperatorio de pacientes críticos sometidos a cirugía no cardiaca, influye en las decisiones anestésicas. Con una indicación juiciosa y adecuada es útil y permite un manejo fisiopatológico exacto de estos enfermos. Se trata de la técnica de imágenes cardíacas más importante disponible para los anestesiólogos en la actualidad, pero necesariamente deben establecerse criterios de indicaciones que justifiquen su utilización, para lograr sus beneficios.

\section{Referencias}

1. Swan HJ, Ganz W, Forrester J, Marcus $\mathrm{H}$, Diamond G, Chonette $D$. Catheterization of the heart in man with use of a flow-directed balloon-tipped catheter. N Engl J Med 1970 Aug;283(9):447-51.

2. American Society of Anesthesiologists and Society of Cardiovascular Anesthesiologists Task Force on Transesophageal Echocardiography. Practice Guidelines for perioperative transesophageal Echocardiography. Anesthesiology 2010 May;112(5):108496.

3. Osman D, Ridel C, Ray P, Monnet $X$, Anguel N, Richard $C$ et al. Cardiac filling pressures are not appropriate to predict hemodynamic response to volume challenge. Crit Care Med 2007 Jan;35(1):64-8.

4. Reeves ST, Finley AC, Skubas NJ, Swaminathan M, Whitley WS, Glas KE et al.; Council on Perioperative Echocardiography of the American Society of Echocardiography and the Society of Cardiovascular Anesthesiologists. Special article: basic perioperative transesophageal echocardiography examination: a consensus statement of the American Society of Echocardiography and the Society of Cardiovascular Anesthesiologists. Anesth Analg 2013 Sep;117(3):543-58.

5. Marik PE, Baram M, Vahid B.
Does central venous pressure predict fluid responsiveness? A systematic review of the literature and the tale of seven mares. Chest 2008 Jul;134(1):172-8.

6. Patteril M, Swaminathan M. Pro: intraoperative transesophageal echocardiography is of utility in patients at high risk of adverse cardiac events undergoing noncardiac surgery. J Cardiothorac Vasc Anesth 2004 Feb;18(1):107-9.

7. Suriani RJ, Neustein S, ShoreLesserson L, Konstadt S. Intraoperative transesophageal echocardiography during noncardiac surgery. J Cardiothorac Vasc Anesth 1998 Jun;12(3):274-80.

8. Schulmeyer MC, Santelices E, 
Vega R, Schmied S. Impact of intraoperative transesophageal echocardiography during noncardiac surgery. J Cardiothorac Vasc Anesth 2006 Dec;20(6):768-71.

9. Cabrera Schulmeyer MC, Santelices E, Vega R. Ecocardiografía Transesofágica en cirugía no cardíaca. Rev Col Anestesia 2004;32:29-34.

10. Kolev N, Brase R, Swanevelder J, Oppizzi M, Riesgo MJ, van der Maaten JM et al.; European Perioperative TOE Research Group. The influence of transoesophageal echocardiography on intra-operative decision making. A European multicentre study. Anaesthesia 1998 Aug;53(8):767-73.

11. Cahalan MK, Stewart W, Pearlman A, Goldman M, SearsRogan P, Abel M et al.; Society of Cardiovascular Anesthesiologists; American Society of Echocardiography Task Force. American Society of Echocardiography and Society of Cardiovascular Anesthesiologists task force guidelines for training in perioperative echocardiography. J Am Soc Echocardiogr 2002 Jun;15(6):647-52.

12. Jensen $M B$, Sloth $E$, Larsen $K M$, Schmidt MB. Transthoracic echocardiography for cardiopulmonary monitoring in intensive care. Eur J Anaesthesiol 2004 Sep;21(9):700-7.
13. Swanevelder J, Chin D, Kneeshaw J, Chambers J, Bennett S, Smith $D$ et al. Accreditation in transoesophageal echocardiography: statement from the Association of Cardiothoracic Anaesthetists and the British Society of Echocardiography Joint TOE Accreditation Committee. $\mathrm{Br}$ J Anaesth 2003 Oct;91(4):46972.

14. Aronson S, Thys DM. Training and certification in perioperative transesophageal echocardiography: a historical perspective. Anesth Analg 2001 Dec;93(6):1422-7.

15. Kneeshaw JD. Transoesophageal echocardiography (TOE) in the operating room. Br J Anaesth 2006 Jul;97(1):77-84.

16. Mahmood F, Christie A, Matyal R. Transesophageal echocardiography and noncardiac surgery. Semin Cardiothorac Vasc Anesth 2008 Dec;12(4):265-89.

17. Bouchard MJ, Denault A, Couture P, Guertin MC, Babin D, Ouellet $P$ et al. Poor correlation between hemodynamic and echocardiographic indexes of left ventricular performance in the operating room and intensive care unit. Crit Care Med 2004 Mar;32(3):644-8.

18. Catena E, Mele D. Role of intraoperative transesophageal echocardiography in patients undergoing noncardiac surgery. J Cardiovasc Med (Hagerstown)
2008 Oct;9(10):993-1003.

19. Salem $R$, Denault $A Y$, Couture P, Bélisle S, Fortier A, Guertin $M C$ et al. Left ventricular enddiastolic pressure is a predictor of mortality in cardiac surgery independently of left ventricular ejection fraction. $\mathrm{Br} J$ Anaesth 2006 Sep;97(3):292-7.

20. Djaiani GN, Shernan SK. Intraoperative assessment of diastolic function: utility of echocardiography. Curr Opin Anaesthesiol 2003 Feb; 16(1):11-9.

21. Royse C. Ultrasound-guided haemodynamic state assessment. Best Practice Clinical research anesthesiology 2009: 273-283.

22. Cabrera C, Farías J, Rajdl E. Utility of transesophageal echocardiography during severe hypotension in non-cardiac surgery. Rev Bras Anestesiolo; 2010. p. 60.

23. Cabrera Schulmeyer MC, Schmied S, Vega R, Semertzakis I, De la Maza J. Intraoperative diastolic disfunction as a risk factor of postoperative hemodynamic disfunction. Rev Med Chile 2007;135:1276-81.

24. Cabrera Schulmeyer MC, Labbé M, Schmied S, De la Maza J. Ecocardiografía transesofágica intraoperatoria y movimiento sistólico anterior de válvula mitral: hallazgo intraoperatorio durante cirugía no cardíaca. Rev Esp Anestesiol Reanim 2007 Aug-Sep;54(7):440-3. 